Published in "Physical Review A - Atomic, Molecular and Optical Physics

84(2): 022502, 2011"

which should be cited to refer to this work.

\title{
Spectroscopy of barium atoms in liquid and solid helium matrices
}

\author{
V. Lebedev, ${ }^{*}$ P. Moroshkin, and A. Weis \\ Département de Physique, Université de Fribourg, Chemin du Musée 3, CH-1700 Fribourg, Switzerland
}

\begin{abstract}
We present an exhaustive overview of optical absorption and laser-induced fluorescence lines of $\mathrm{Ba}$ atoms in liquid and solid helium matrices in visible and near-infrared spectral ranges. Due to the increased density of isolated atoms, we have found a large number of spectral lines that were not observed in condensed helium matrices before. We have also measured the lifetimes of metastable states. The lowest ${ }^{3} D_{1}$ metastable state has lifetime of $2.6 \mathrm{~s}$ and can be used as an intermediate state in two-step excitations of high-lying states. Various matrix-induced radiationless population transfer channels have been identified.
\end{abstract}

\section{INTRODUCTION}

The spectroscopy of foreign atoms and molecules in liquid and solid ${ }^{4} \mathrm{He}$ matrices has been developed since the $1980 \mathrm{~s}$ and was recently reviewed by Moroshkin et al. [1]. Besides the heavier alkali metals ( $\mathrm{Cs}$ and $\mathrm{Rb}$ ), barium is the most well-studied dopant that has been investigated by research groups in Paris, Heidelberg, Saitama, Kyoto, and Garching. In the pioneering spectroscopic experiments [2,3] on atoms in quantum matrices, the $6{ }^{2} S_{1 / 2}-6{ }^{2} P_{1 / 2,3 / 2}$ resonance transitions of positively charged $\mathrm{Ba}^{+}$ions were observed. In those experiments the ions were introduced into liquid helium by an electric-field-driven flow from an ionization source placed above the liquid surface.

Later this implantation technique was extended to neutral dopants [4-6]. Electronically excited $\mathrm{Ba}$ atoms at densities of $\approx 10^{8} \mathrm{~cm}^{-3}$ were produced by the recombination of implanted $\mathrm{Ba}^{+}$ions with free electrons in the liquid helium bath, and the spectrum of the recombination-induced fluorescence was observed. Larger dopant densities (up to $10^{10} \mathrm{~cm}^{-3}$ ) were obtained by producing $\mathrm{Ba}$ atoms via direct laser ablation in liquid helium, close to the observation zone [7-10]. Those studies focused on the laser-induced fluorescence (LIF) and excitation spectra of the singlet $6 s^{2}-6 s 6 p$ transition and of the triplet $5 d 6 s-5 d 6 p$ transitions whose lowest state is the metastable $5 d 6 s^{3} D_{1}$. It was first shown by Bauer et al. [5] that this metastable state acquires a significant steady-state population in liquid helium matrices.

The first study of laser excitation and fluorescence spectra of Ba atoms in solid helium was carried out by Kanorsky et al. $[10,11]$ in Garching. The density of implanted atoms in those experiments was estimated to be $\approx 10^{10} \mathrm{~cm}^{-3}$. Their spectroscopic studies focused on the (strongest) singlet transition $\left(6 s^{2}-6 s 6 p\right)$ at $18115 \mathrm{~cm}^{-1}$, for which the dependence of the excitation and emission line shapes on the helium pressure were measured and explained.

In order to interpret the observed spectral shifts and widths, the atomic bubble model, an extension of the electron bubble model [12], was developed and refined [5,8,11,13,14]. Calculations were carried out for the $6 s^{2}-6 s 6 p$ singlet transition and yielded line shapes in good agreement with experimental findings.

\footnotetext{
*victor.lebedev@unifr.ch
}

The experiments reported here were triggered by our recent discovery that positively charged impurity ions play an important role in the nucleation of helium crystals and for the stabilization of the doped helium crystal against melting $[15,16]$. It was demonstrated that laser ablation of alkali metals in liquid and solid helium produces a large number of positively charged ions that become isolated in the matrix. Due to electrostriction these ions form snowballs that act as nucleation centers for the solidification of liquid He [16] and stabilize the crystal below the melting pressure [15]. Similar impurity-stabilized solids ("icebergs") have been observed in barium-doped condensed helium [17]. The study of absorption and fluorescence spectra of $\mathrm{Ba}^{+}$ions trapped in solid helium and in the "iceberg" would provide accurate quantitative information on their density and distribution in the sample, a prerequisite for a quantitative understanding of these spectacular phenomena.

To our big surprise we were not able to identify a single spectral feature (in the visible and near infrared domains) that can be unambiguously assigned to $\mathrm{Ba}^{+}$transition. In searching for $\mathrm{Ba}^{+}$lines we have carried out a detailed spectroscopic study of the matrices doped by laser ablation of Ba metal. Our experiments cover a broad spectral range $\left(8000-25000 \mathrm{~cm}^{-1}\right)$. The greatly enhanced density of implanted atoms has allowed us to detect a large number of atomic lines, thereby doubling the number of identified spectral lines of the $\mathrm{Ba}$ atom in such matrices.

\section{EXPERIMENTAL SETUP}

The experiment was carried out in a setup described in detail in [1]. The helium host matrix is prepared in a high-pressure cell cooled by a helium bath in a cryostat with optical access along five orthogonal directions. The helium pressure in the cell can be varied from 1 to $40 \mathrm{bar}$, and all measurements were carried out at $1.5 \mathrm{~K}$. Implantation of $\mathrm{Ba}$ atoms and clusters was done by means of laser ablation from a Ba metal target mounted at the bottom of the cell. The second harmonic radiation from a pulsed Nd:YAG laser (up to $100 \mathrm{~mJ}$ per pulse) was admitted from the top and focused on the target by a lens mounted above the cell.

In liquid helium the ablated atoms quickly diffuse out of the observation region, so that measurements in the liquid phase were performed under continuous full power ablation using pulses with a repetition rate of $5-10 \mathrm{~Hz}$. 


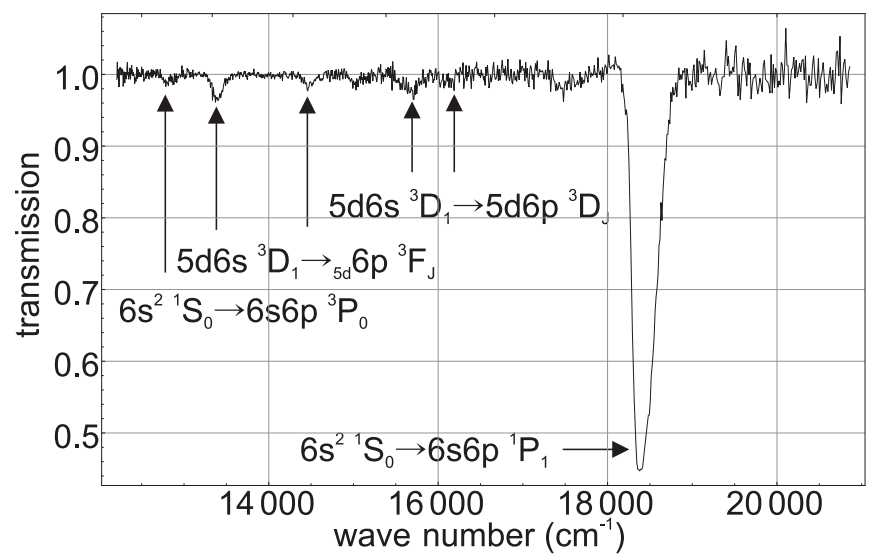

FIG. 1. Transmission spectrum of a barium-doped helium crystal.

Doped solid samples were produced by several hundred pulses of the ablation laser at a rate of 1-2 Hz. The slow diffusion of the $\mathrm{Ba}$ atoms in the solid He matrix leads to the formation of clusters and nanoparticles, as witnessed by the decrease of the atomic fluorescence signal on a time scale of hours. For comparison, the cluster formation in alkali-metal (Cs, Rb)-doped crystals occurs within minutes under similar conditions. In order to maintain a constant density of trapped atoms we photodissociate the clusters by $30 \mathrm{~mJ}$ pulses of the same Nd:YAG laser fired every second.

The achieved steady-state density of Ba atoms in the solid sample is several orders of magnitude larger than in all previous experiments. The large density allows a detection of the resonance by transmission spectroscopy. We pass a beam from a halogen lamp (color temperature of $3300 \mathrm{~K}$ ) through the sample and disperse the transmitted light by a grating spectrometer. A typical transmission spectrum is shown in Fig. 1. For comparison, we recall that the much smaller steady-state density in alkali-metal-doped crystals yield absorptions of only a few times $10^{-5}$ [18].

When observed from a direction orthogonal to the illumination beam, the doped part of the crystal has a gray color due to light scattering by defects (Fig. 2). In the central part of the doped region, the atom production by laser-induced breakup of

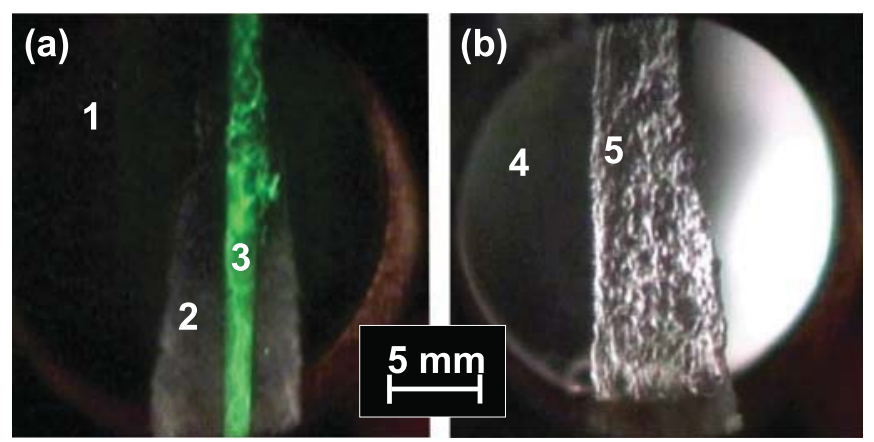

FIG. 2. (Color online) Ba-doped helium crystals as seen through a cryostat windows before (a) and after (b) melting of pure He: 1, pure solid helium; 2, doped helium crystal; 3 , region containing Ba atoms as evidenced by the green resonance fluorescence induced by lateral illumination with white light; 4, liquid helium; 5, impurity-stabilized doped helium crystal (iceberg). clusters is most efficient, and the doped column emits intense continuous green $\left(18060 \mathrm{~cm}^{-1}\right)$ fluorescence that is visible by the naked eye.

In the experiments reported below we used radiation from the second and third harmonics of a pulsed Nd:YAG laser, as well as the signal and idler beams of a tunable optical parametric oscillator (OPO) pumped by the third harmonic of a second pulsed Nd:YAG laser. We used either a single beam of one of the mentioned lasers or a combination thereof. Fluorescence was collected at $90^{\circ}$ and analyzed by a grating spectrograph equipped with a CCD camera (resolution $\approx 5 \mathrm{~cm}^{-1}$ ). Excitation spectra were recorded by scanning the OPO wavelength and monitoring the fluorescence intensity induced by the signal or idler beam. The resolution of the excitation spectra is limited by the OPO linewidth and varies from $\approx 40 \mathrm{~cm}^{-1}$ in the $25000-14300 \mathrm{~cm}^{-1}$ range (signal output) to $\approx 50 \mathrm{~cm}^{-1}$ in the $12800-10000 \mathrm{~cm}^{-1}$ region (idler output). The numerical values of the fluorescence linewidths given below have been corrected for the spectrometer resolution.

\section{RESULTS AND DISCUSSION}

In agreement with earlier observations [17], we found that Ba-doped condensed helium forms an impurity-stabilized solid [iceberg, Fig. 2(b)], similar to the one of alkali-metaldoped He [15]. By measuring the laser-induced electric current (technique described in [16]) we have proven the presence of a large amount of charged particles ( $\mathrm{Ba}^{+}$ions and free electrons) produced by the laser ablation. However, we could not detect any fluorescence from $\mathrm{Ba}^{+}$ions either in superfluid (1.6 bar) or in solid (28-38 bar) He in the range of $8000-25000 \mathrm{~cm}^{-1}$. The absorption spectrum of $\mathrm{Ba}^{+}$in unpressurized superfluid $\mathrm{He}$ [3] consists of the two $6{ }^{2} S_{1 / 2}-6{ }^{2} P_{1 / 2,3 / 2}$ broad lines centered at 22730 and $20830 \mathrm{~cm}^{-1}$. The corresponding fluorescence lines are centered at 20400 and $19050 \mathrm{~cm}^{-1}$, respectively [3]. In our experiments in solid He, scans of the OPO wavelength in the range of $20000-23250 \mathrm{~cm}^{-1}$ produced only fluorescence from neutral $\mathrm{Ba}$ and $\mathrm{Sr}$ atoms, the latter being present as a $10^{-4}$ contamination in the Ba ablation target.

We are left completely puzzled by the fact that we observe spectra from neither $\mathrm{Ba}^{+}$nor $\mathrm{Ba}^{+}$exciplexes [19] in solid $\mathrm{He}$ and in pressurized (1.6 bar) He II.

\section{A. Fluorescence spectra}

The relevant energy levels and electronic transitions in the free barium atom are shown in Fig. 3. All energies are given with respect to the $6 s^{2}{ }^{1} S_{0}$ ground state. The ionization threshold is at $42035 \mathrm{~cm}^{-1}$.

The high-power radiation of the frequency-tripled Nd:YAG laser at $28195 \mathrm{~cm}^{-1}$ populates a large number of excited states via cascades of radiative and radiationless transitions. Figure 4 gives an overview of all fluorescence transitions that can be observed in condensed He following excitation by the pulsed ultraviolet radiation. Our assignment of the spectral lines to $\mathrm{Ba}$ atomic transitions, as well as their shifts with respect to the wavelengths in the free $\mathrm{Ba}$ atom and their full widths at half maximum (FWHM) are listed in Table I. 


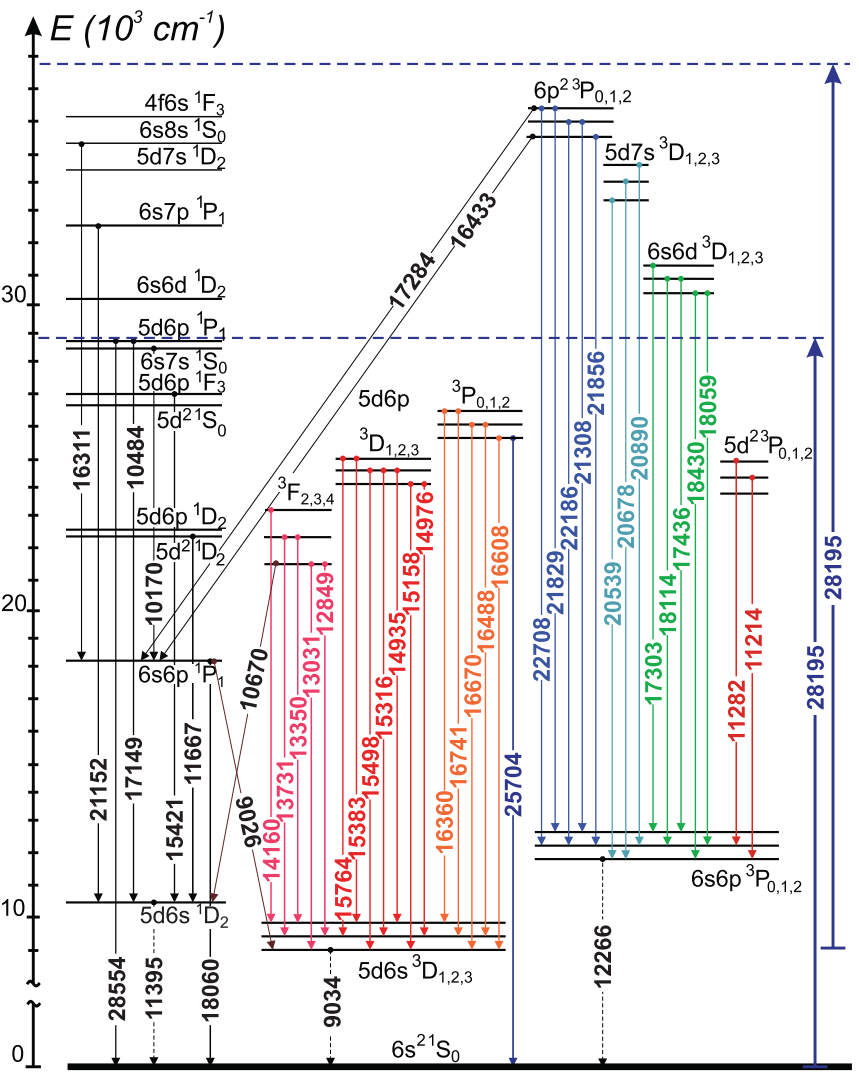

FIG. 3. (Color online) Energy levels of the free barium atom. Arrows indicate optical transitions with their energies given in wave numbers. Solid and dashed arrows correspond to electric dipole allowed and forbidden transitions, respectively. Note that the actual term energies and transition wavelengths in solid and liquid He differ from those of the free $\mathrm{Ba}$ atom as discussed in the text. Horizontal dashed lines mark the energy that can be reached with one photon of the third harmonic of the Nd:YAG laser from the ground $6 s^{2}{ }^{1} S_{0}$ state (lower line) and from the lowest metastable $5 d 6 s^{3} D_{1}$ state (upper line).

Pronounced differences are found in the fluorescence spectra of $\mathrm{Ba}$ atoms in low-pressure (1.6 bar) superfluid helium and in solid helium at 30 bar. Most of the spectral lines in solid He are several nanometers broad and blueshifted with respect to their positions in the free Ba atom. In general, spectra recorded in the liquid matrix have lower intensities than those from solid matrices, a consequence of the much lower atomic density achieved in liquid $\mathrm{He}$ due to diffusion and cluster formation. The line shapes in liquid He have smaller widths (about $10-50 \mathrm{~cm}^{-1}$ FWHM, or resolution limited) and their wavelengths are closer to those in the free atom.

\section{Fluorescence from the singlet $6 \mathrm{~s} 6 \mathrm{p}^{1} P_{1}$ state}

The strongest fluorescence line (Fig. 2) at $18115 \mathrm{~cm}^{-1}$ corresponds to the transition from the $6 s 6 p{ }^{1} P_{1}$ state to the ground $6 s^{2}{ }^{1} S_{0}$ state. The dependence of its spectrum (shown in Fig. 5) on helium pressure is discussed in detail below (Sec. III B). We also observe a much weaker singlet-triplet transition from the $6 s 6 p{ }^{1} P_{1}$ state to the $5 d 6 s{ }^{3} D_{1}$ state at $9075 \mathrm{~cm}^{-1}$.

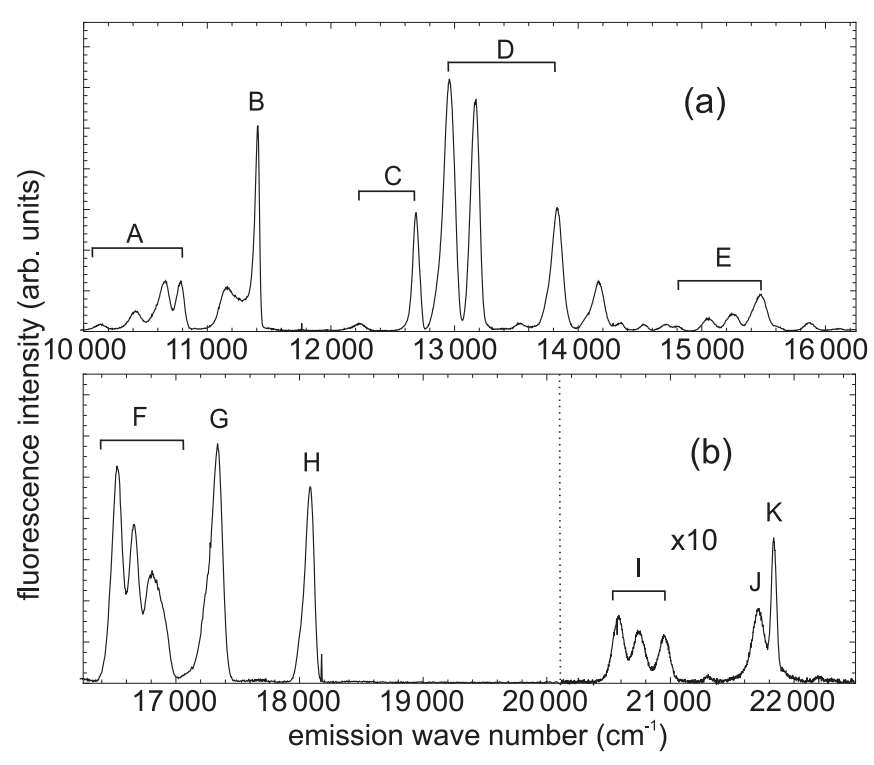

FIG. 4. Spectrum of fluorescence of atomic Ba in solid helium (31 bar, $1.5 \mathrm{~K}$ ) after excitation by pulsed radiation at $28195 \mathrm{~cm}^{-1}$. A, G, unidentified; B, $6 s^{2}{ }^{1} S_{0}-5 d 6 s{ }^{1} D_{2}$; C, $6 s^{2}{ }^{1} S_{0}-6 s 6 p{ }^{3} P_{J}$; D, $5 d 6 s^{3} D_{J}-5 d 6 p^{3} F_{J}$; E, $5 d 6 s^{3} D_{J}-5 d 6 p^{3} D_{J}$; F, $5 d 6 s^{3} D_{J}-$ $5 d 6 p^{3} P_{J} ; \mathrm{H}, 6 s^{2}{ }^{1} S_{0}-6 s 6 p^{1} P_{1} ; \mathrm{I}, 6 s 6 p^{3} P_{J}-5 d 7 s^{3} D_{J} ; \mathrm{J}$, $5 s^{2}{ }^{1} S_{0}-5 s 5 p{ }^{1} P_{1}$ of $\mathrm{Sr} ; \mathrm{K}, 6 s 6 p^{3} P_{J}-6 p^{2}{ }^{3} P_{J}$.

\section{Fluorescence from metastable states}

We have identified three groups of metastable states: $5 d 6 s^{1} D_{2}$ at $11395 \mathrm{~cm}^{-1}, 5 d 6 s^{3} D_{1,2,3}$ at $\approx 9000 \mathrm{~cm}^{-1}$, and $6 s 6 p^{3} P_{0,1,2}$ at $\approx 13000 \mathrm{~cm}^{-1}$ (Fig. 3). Radiative transitions from those states toward the ground state are forbidden in the free $\mathrm{Ba}$ atom. Nevertheless, we could observe their fluorescence in condensed $\mathrm{He}$ at 11 400, 9000, and $12640 \mathrm{~cm}^{-1}$, respectively (Fig. 4; Table I). We have investigated the lifetime of the $6 s 6 p^{3} P_{1}$ state and observed the excitation spectrum of the $6 s^{2}{ }^{1} S_{0}-6 s 6 p^{3} P_{1}$ transition (discussed in Sec. III C). The fluorescence and excitation spectra of the $6 s^{2}{ }^{1} S_{0}-5 d 6 s{ }^{1} D_{2}$ transition are discussed in the same section. The $5 d 6 s^{3} D_{1}$ state is particularly long-lived (discussed in Sec. IIID). It accumulates a large steady-state population and thus serves as a ground state for a number of excitation transitions toward higher-lying triplet states, as discussed in Sec. III E.

\section{Fluorescence from the $5 d 6 p$ configuration}

We have identified numerous transitions from the ${ }^{3} P_{J},{ }^{3} D_{J}$, and ${ }^{3} F_{J}$ states of the configuration $5 d 6 p$ to the $5 d 6 s{ }^{3} D_{J}$ states that dominate the spectral range between $17000 \mathrm{~cm}^{-1}$ and $13000 \mathrm{~cm}^{-1}$ (Fig. 4). These excitation spectra are discussed in Sec. III E.

\section{Fluorescence from the $5 d 7 s^{3} D_{J}$ states}

We tentatively assign weak lines in the $20500-21000 \mathrm{~cm}^{-1}$ range to transitions from the highly excited $5 d 7 s^{3} D_{1,2,3}$ states to the $6 s 6 p^{3} P_{1,2}$ states. Those lines could be observed only under excitation by the third harmonic of the Nd:YAG laser in solid $\mathrm{He}$ and are one order of magnitude weaker than the lines described above. 
TABLE I. Fluorescence lines of Ba observed in a solid helium matrix $(31$ bar, $1.5 \mathrm{~K})$ after excitation by pulsed radiation at $28195 \mathrm{~cm}^{-1}$. Superscripts a, b, c, and d denote lines observed earlier in superfluid helium in [4,5,7], and [8], respectively.

\begin{tabular}{|c|c|c|c|c|c|}
\hline & & \multicolumn{2}{|c|}{ Free atom } & \multicolumn{2}{|c|}{$\begin{array}{l}\text { In solid } \mathrm{He} \\
\left(\mathrm{cm}^{-1}\right)\end{array}$} \\
\hline \multicolumn{2}{|c|}{ Transition } & $\left(\mathrm{cm}^{-1}\right)$ & $(\mathrm{nm})$ & Shift & Width \\
\hline $6 s 6 p-5 d 6 s$ & ${ }^{1} P_{1}-{ }^{3} D_{1}$ & 9026 & 1102 & +15 & 45 \\
\hline $6 s 6 p-6 s^{2}$ & $\begin{array}{l}{ }^{1} P_{1}-{ }^{1} S_{0} \mathrm{a}, \mathrm{b}, \mathrm{c}, \mathrm{d} \\
{ }^{3} P_{1}-{ }^{1} S_{0}\end{array}$ & $\begin{array}{l}18060 \\
12637\end{array}$ & $\begin{array}{l}554 \\
791\end{array}$ & $\begin{array}{l}+30 \\
+35\end{array}$ & $\begin{array}{l}80 \\
60\end{array}$ \\
\hline $5 d 6 p-5 d 6 s$ & $\begin{array}{l}{ }^{3} F_{2}-{ }^{3} D_{1}{ }^{\mathrm{a}} \\
{ }^{3} F_{2}-{ }^{3} D_{2}^{\mathrm{a}} \\
{ }^{3} F_{3}-{ }^{3} D_{2} \\
{ }^{3} F_{3}-{ }^{3} D_{3} \\
{ }^{3} D_{1}-{ }^{3} D_{1}^{\mathrm{a}} \\
{ }^{3} D_{1}-{ }^{3} D_{2}{ }^{\mathrm{a}} \\
{ }^{3} D_{2}-{ }^{3} D_{1}^{\mathrm{a}} \\
{ }^{3} D_{2}-{ }^{3} D_{2}{ }^{\mathrm{a}} \\
{ }^{3} D_{2}-{ }^{3} D_{3} \\
{ }^{3} D_{3}-{ }^{3} D_{2}{ }^{\mathrm{a}} \\
{ }^{3} D_{3}-{ }^{3} D_{3}^{\mathrm{a}} \\
{ }^{3} P_{0}-{ }^{3} D_{1} \mathrm{a}, \mathrm{b}, \mathrm{c}, \mathrm{d} \\
{ }^{3} P_{1}-{ }^{3} D_{1} \mathrm{a}, \mathrm{c}, \mathrm{d}, \\
{ }^{3} P_{1}-{ }^{3} D_{2}^{\mathrm{a}, \mathrm{b}, \mathrm{c}, \mathrm{d}} \\
{ }^{3} P_{2}-{ }^{3} D_{1} \\
{ }^{3} P_{2}-{ }^{3} D_{2} \mathrm{a,b} \\
{ }^{3} P_{2}-{ }^{3} D_{3}^{\mathrm{a}, \mathrm{b}, \mathrm{d}} \\
{ }^{3} P_{2}-{ }^{1} D_{2} \\
{ }^{3} F_{2}-{ }^{1} D_{2}\end{array}$ & $\begin{array}{l}13031 \\
12849 \\
13731 \\
13350 \\
15158 \\
14976 \\
15498 \\
15316 \\
14935 \\
15764 \\
15383 \\
16608 \\
16670 \\
16488 \\
16923 \\
16741 \\
16360 \\
14562 \\
10670\end{array}$ & $\begin{array}{l}767 \\
778 \\
729 \\
750 \\
660 \\
668 \\
645 \\
653 \\
670 \\
634 \\
650 \\
602 \\
600 \\
606 \\
591 \\
597 \\
611 \\
688 \\
937\end{array}$ & $\begin{array}{r}+140 \\
+110 \\
+100 \\
+40 \\
+100 \\
+100 \\
+100 \\
+110 \\
+100 \\
+100 \\
+100 \\
+85 \\
+80 \\
+70 \\
+40 \\
+75 \\
+65 \\
+65 \\
+130\end{array}$ & $\begin{array}{r}80 \\
100 \\
100 \\
120 \\
120 \\
110 \\
100 \\
90 \\
100 \\
90 \\
90 \\
50 \\
50 \\
50 \\
50 \\
50 \\
50 \\
50 \\
100\end{array}$ \\
\hline $5 d^{2}-6 s 6 p$ & $\begin{array}{l}{ }^{3} P_{0}-{ }^{3} P_{1} \\
{ }^{3} P_{0}-{ }^{3} P_{0} \\
{ }^{3} P_{1}-{ }^{3} P_{2}\end{array}$ & $\begin{array}{c}10572 \\
11043 \\
9965\end{array}$ & $\begin{array}{c}946 \\
905 \\
1004\end{array}$ & $\begin{array}{l}+110 \\
+100 \\
+170\end{array}$ & $\begin{array}{r}85 \\
110 \\
110\end{array}$ \\
\hline $6 s 7 s-6 s 6 p$ & $\begin{array}{l}{ }^{3} S_{1}-{ }^{3} P_{1} \\
{ }^{1} S_{0}-{ }^{1} P_{1}\end{array}$ & $\begin{array}{l}13523 \\
10170\end{array}$ & $\begin{array}{l}740 \\
983\end{array}$ & $\begin{array}{l}+15 \\
-25\end{array}$ & $\begin{array}{l}70 \\
90\end{array}$ \\
\hline $\begin{array}{l}6 s 6 d-6 s 6 p \\
5 d 7 s-6 s 6 p\end{array}$ & $\begin{array}{l}{ }^{1} D_{2}-{ }^{1} P_{1} \\
{ }^{3} D_{1}-{ }^{3} P_{0} \\
{ }^{3} D_{2}-{ }^{3} P_{0} \\
{ }^{3} D_{3}-{ }^{3} P_{1}\end{array}$ & $\begin{array}{l}12177 \\
20539 \\
20678 \\
20890\end{array}$ & $\begin{array}{l}821 \\
487 \\
484 \\
479\end{array}$ & $\begin{array}{l}+70 \\
+40 \\
+65 \\
+60\end{array}$ & $\begin{array}{r}90 \\
90 \\
90 \\
100\end{array}$ \\
\hline $6 p^{2}-6 s 6 p$ & $\begin{array}{l}{ }^{3} P_{1}-{ }^{3} P_{0} \\
{ }^{3} P_{1}-{ }^{3} P_{1} \\
{ }^{3} P_{1}-{ }^{3} P_{2} \\
{ }^{3} P_{2}-{ }^{3} P_{2}\end{array}$ & $\begin{array}{ll}22 & 557 \\
22 & 124 \\
21 & 230 \\
21 & 829\end{array}$ & $\begin{array}{l}443 \\
425 \\
471 \\
458\end{array}$ & $\begin{array}{l}\approx 0 \\
\approx 0 \\
\approx 0 \\
\approx 0\end{array}$ & $\begin{array}{l}40 \\
40 \\
40 \\
50\end{array}$ \\
\hline
\end{tabular}

\section{Fluorescence from the $6 p^{2}{ }^{3} P_{J}$ states}

The group of lines between 21300 and $23000 \mathrm{~cm}^{-1}$ was observed only under excitation by the third harmonic of the $\mathrm{Nd}$ :YAG laser. The number and relative intensities of the lines differ when observed in liquid $\mathrm{He}$ at 1.6 bar or in solid $\mathrm{He}$ at 31 bar. We attribute those lines to transitions between the $6 p^{2}{ }^{3} P_{J}$ states and the metastable $6 s 6 p{ }^{3} P_{J^{\prime}}$ states.

In low-pressure liquid helium, the lines at 21834 and 21231 $\mathrm{cm}^{-1}$ are the brightest in this group. Their intensity ratio suggests an assignment to $(J=0) \rightarrow(J=1)$ and $(J=1) \rightarrow$ $(J=2)$ transitions. Other strong transitions between these multiplets are not present, most probably because the $J=2$ state of the upper multiplet is not populated by the specific excitation process. In solid helium one expects a stronger

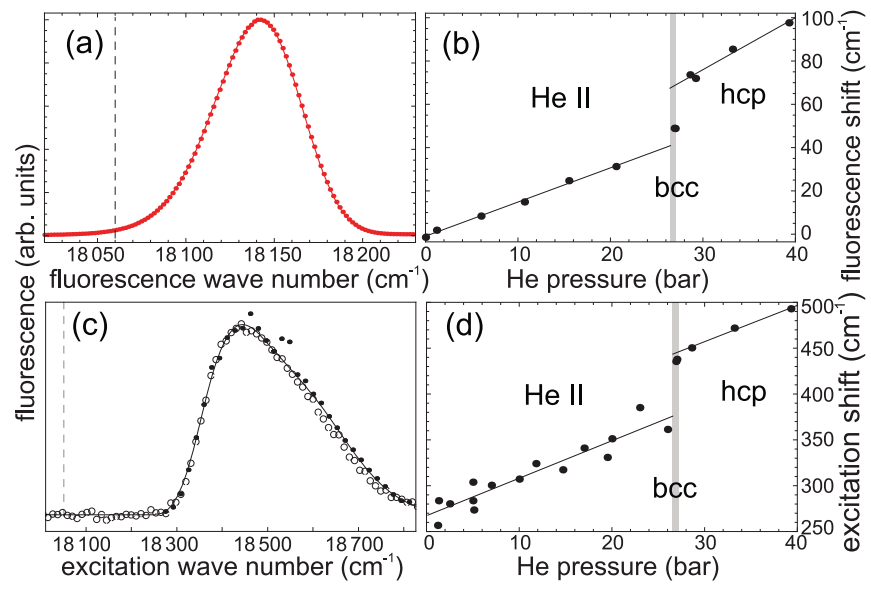

FIG. 5. (Color online) Fluorescence (a) and excitation/absorption (c) spectra of the $6 s^{2}{ }^{1} S_{0}-6 s 6 p{ }^{1} P_{1}$ transition of $\mathrm{Ba}$ in solid He; $p=28.6 \mathrm{bar}, T=1.50 \mathrm{~K}$. Experimental data are shown as dots and the solid line is a guide for the eye. In (c) the open circles represent the absorption coefficient $\kappa(\tilde{v})$ inferred from Fig. 1 (amplitude scaled). The vertical dashed line at $18060 \mathrm{~cm}^{-1}$ marks the transition wavelength in the free $\mathrm{Ba}$ atom. Dependence of the matrix induced shifts of the $6 s^{2}{ }^{1} S_{0}-6 s 6 p{ }^{1} P_{1}$ line on the solid He density in emission (b) and in excitation (d), where dots represent experimental data and solid lines are linear fits. The points for liquid helium in (d) are taken from [10].

perturbation of the atomic levels and their fast radiationless deexcitation toward lower states in the multiplet. We can observe only the $(J=0) \rightarrow(J=1)$ and the much weaker $(J=0) \rightarrow(J=0)$ transitions. Our experiments could not detect any fluorescence from states lying above the $6 p^{2}{ }^{3} P_{1}$ state, located at $34823 \mathrm{~cm}^{-1}$ (in the free atom), that can be reached by single photon absorption at $28195 \mathrm{~cm}^{-1}$ from the lowest metastable state.

\section{Fluorescence from the Sr atom}

We have also observed the strongest fluorescence line $\left(5 s 5 p{ }^{1} P_{1}-5 s^{2}{ }^{1} S_{0}\right.$ transition at $\left.21750 \mathrm{~cm}^{-1}\right)$ of strontium, which is the dominant contamination of our barium ablation target. Spectra of fluorescence and resonant excitation of this line recorded in solid helium are similar to those obtained in [5] in liquid He.

\section{B. Resonance transition $6 s^{2}{ }^{1} S_{0}-6 s 6 p^{1} P_{1}$}

The resonance line $6 s^{2}{ }^{1} S_{0} \rightleftarrows 6 s 6 p^{1} P_{1}\left(18060 \mathrm{~cm}^{-1}\right.$ in the free atom) is the strongest and best studied spectral line of $\mathrm{Ba}$ in helium matrices. Its broadening and matrix-induced shift in liquid and solid helium have been measured and modeled in the past $[4,11]$. The broadening and shift of the line are much more pronounced in excitation than in fluorescence spectra (Fig. 5). In agreement with the earlier results, we found a linear dependence of the line shift [Figs. 5(b) and 5(d)] of that resonance line in solid $\mathrm{He}$ on He pressure. The line center of the fluorescence spectrum was found to shift at a rate of $2.2 \mathrm{~cm}^{-1} /$ bar, which is larger than the rate of $1.54 \mathrm{~cm}^{-1} /$ bar reported in [11]. The excitation spectrum shifts at a rate of $4.0 \mathrm{~cm}^{-1} /$ bar, which is comparable to the $3.8 \mathrm{~cm}^{-1} /$ bar reported in [11]. 
As can be seen from Figs. 5(b) and 5(d), there is a discontinuity in the shift of the fluorescence line at the phase boundary between the hexagonal close-packed (hcp) and body-centered cubic (bcc) crystalline phases of He at 26.7 bar. A similar discontinuity was observed earlier in experiments with atomic cesium in solid helium and successfully explained in the frame of the atomic bubble model [20].

As reported in Sec. II, the high-density of trapped $\mathrm{Ba}$ atoms allowed us to observe the $6 s^{2}{ }^{1} S_{0}-6 s 6 p{ }^{1} P_{1}$ transition directly by transmission spectroscopy (Fig. 1). The optical thickness $\kappa(\tilde{v}) L=-\ln T(\tilde{v})$ at $18500 \mathrm{~cm}^{-1}$ reaches 0.60 , and the absorption line shape matches very well the excitation spectrum recorded via LIF, as shown in Fig. 5(c).

Based on the transmission spectrum we have estimated the density of the $\mathrm{Ba}$ atoms in the ground state:

$$
N_{i}=\frac{m_{0} c}{\pi e^{2}} \frac{1}{f_{i k}} \frac{1}{L} \int \frac{-\ln T(\tilde{v})}{\tilde{v}^{2}} d \tilde{v}
$$

where $T(\tilde{v})$ is the transmission spectrum, $L$ is the doped sample thickness (estimated to be $2 \mathrm{~mm}$ from Fig. 2), $m_{0}$ is the electron mass, $c$ is the speed of light, $e$ is the elementary charge, and $f_{i k}=1.64$ is the oscillator strength of the transition taken from $[21,22]$ for the free $\mathrm{Ba}$ atom. This analysis yields a density of $N_{i}=1.0(1) \times 10^{15} \mathrm{~cm}^{-3}$, which is five orders of magnitude larger than the estimated densities of $\mathrm{Ba}$ atoms in solid $\mathrm{He}$ in earlier experiments, and the densities of alkali-metal atoms implanted in solid He using the same setup and implantation technique [1].

\section{Transitions to the quasi-metastable states $5 d 6 s{ }^{1} D_{2}$ and $6 s 6 p^{3} P_{0,1,2}$}

The fluorescence and excitation spectra of the $5 d 6 s{ }^{1} D_{2}$ and $6 s 6 p^{3} P_{0,1}$ states are shown in Fig. 6.

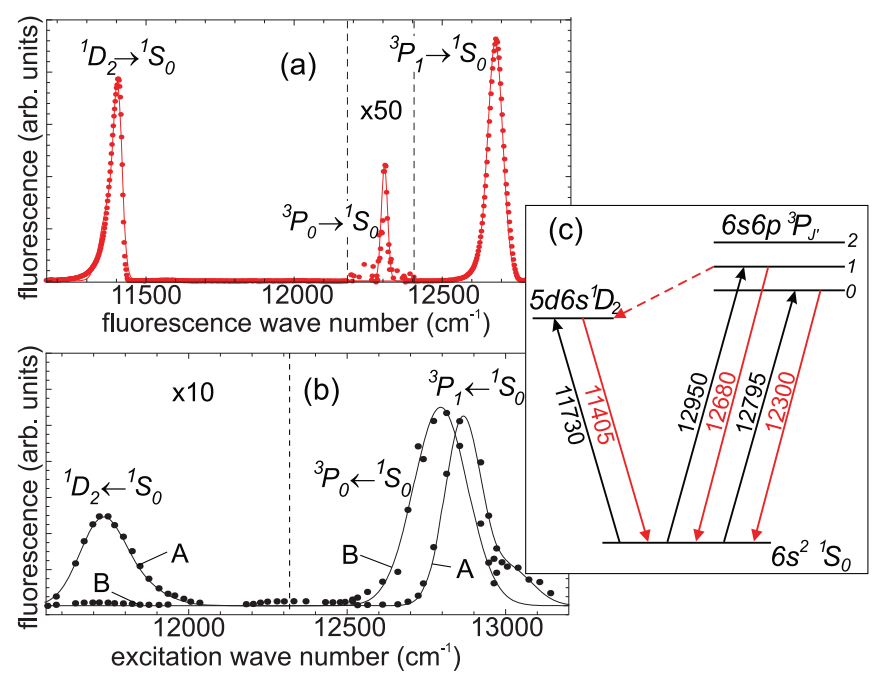

FIG. 6. (Color online) (a) Spectrum of the fluorescence from the quasi-metastable states $6 s 6 p{ }^{3} P_{0,1}$ and $6 s 5 d{ }^{1} D_{2}$ toward the ground $6 s^{2}{ }^{1} S_{0}$ state. Excitation laser at $12950 \mathrm{~cm}^{-1}, p=30$ bar, $T=$ $1.5 \mathrm{~K}$. (b) Excitation spectra of the $6 s^{2}{ }^{1} S_{0}-6 s 6 p^{3} P_{0,1}, 6 s 5 d{ }^{1} D_{2}$ transitions under the same conditions as (a). Curve A, fluorescence yield at $11405 \mathrm{~cm}^{-1}$; curve $\mathrm{B}$, fluorescence yield at $12300 \mathrm{~cm}^{-1}$. (c) Relevant part of the energy diagram. Indicated wave numbers correspond to actual transitions in solid helium.
TABLE II. Wave numbers and wavelengths of excitation lines of $\mathrm{Ba}$ in solid helium at 31 bar, $1.5 \mathrm{~K}$. Superscripts a, b, and c denote the lines observed earlier in superfluid helium $[7,8]$ and in solid helium [10], respectively.

\begin{tabular}{|c|c|c|c|c|c|}
\hline & & \multicolumn{2}{|c|}{ Free atom } & \multicolumn{2}{|c|}{$\begin{array}{l}\text { In solid He } \\
\left(\mathrm{cm}^{-1}\right)\end{array}$} \\
\hline \multicolumn{2}{|c|}{ Transition } & $\left(\mathrm{cm}^{-1}\right)$ & $(\mathrm{nm})$ & Shift & Width \\
\hline \multirow[t]{2}{*}{$6 s 6 p-6 s^{2}$} & ${ }^{1} P_{1}-{ }^{1} S_{0} \mathrm{a}, \mathrm{b}, \mathrm{c}$ & 18060 & 554 & +450 & 300 \\
\hline & ${ }^{3} P_{1}-{ }^{1} S_{0}$ & 12637 & 791 & +160 & 150 \\
\hline \multirow[t]{8}{*}{$5 d 6 p-5 d 6 s$} & ${ }^{3} F_{3}-{ }^{3} D_{1}$ & 13913 & 719 & +520 & 150 \\
\hline & ${ }^{3} F_{2}-{ }^{3} D_{1}$ & 13031 & 767 & +400 & 150 \\
\hline & ${ }^{3} D_{1}-{ }^{3} D_{1}$ & 15158 & 660 & +570 & 250 \\
\hline & ${ }^{3} D_{2}-{ }^{3} D_{1}$ & 15498 & 645 & +660 & 250 \\
\hline & ${ }^{3} D_{3}-{ }^{3} D_{1}$ & 15946 & 627 & +660 & 200 \\
\hline & ${ }^{3} P_{0}-{ }^{3} D_{1}{ }^{\mathrm{a}}$ & 16608 & 602 & +950 & 300 \\
\hline & ${ }^{3} P_{1}-{ }^{3} D_{1}{ }^{\mathrm{a}, \mathrm{b}}$ & 16670 & 600 & +930 & 300 \\
\hline & ${ }^{3} P_{2}-{ }^{3} D_{1}{ }^{\mathrm{a}, \mathrm{b}}$ & 16923 & 591 & +900 & 200 \\
\hline
\end{tabular}

The $6 s 6 p{ }^{3} P_{1}-6 s^{2}{ }^{1} S_{0}$ fluorescence line is centered at $12680 \mathrm{~cm}^{-1}$ both in liquid and in solid He, and is $\approx 50 \mathrm{~cm}^{-1}$ shifted with respect to the (forbidden) free-atomic line. The $6 s 6 p^{3} P_{0}-6 s^{2}{ }^{1} S_{0}$ fluorescence line is centered at $12300 \mathrm{~cm}^{-1}$ and is blueshifted by $35 \mathrm{~cm}^{-1}$ with respect to the corresponding free-atomic line. It is much weaker and can be observed only in the solid He matrix.

The line shapes of these fluorescence lines [Fig. 6(a)] are slightly asymmetric, showing an extended red wing. In solid He at 30 bar, they are $\approx 60 \mathrm{~cm}^{-1}$ broad. As usual, the excitation lines show a larger shift and broadening than the fluorescence lines (Table II).

We have measured the decay time of the stronger $6 s 6 p{ }^{3} P_{1}-6 s^{2}{ }^{1} S_{0}$ line by recording the time-resolved fluorescence signal at the relevant wavelength using a photodiode mounted at the output of the spectrograph. The obtained lifetime of the $6 s 6 p^{3} P_{1}$ state is found to be $\tau=1.20(1) \mu \mathrm{s}$, which is $10 \%$ smaller than in the free atom $\tau=1.345(14) \mu \mathrm{s}$ [23].

The resonant quadrupole transition between the ground state and the singlet quasi-metastable state $5 d 6 s^{1} D_{2}$ shows a similar behavior. The fluorescence line has a more pronounced red wing asymmetry and its maximum intensity has no measurable shift with respect to the $11395 \mathrm{~cm}^{-1}$ energy of the transition in the free atom. The corresponding excitation line, however, is blueshifted by $\approx 330 \mathrm{~cm}^{-1}$ and has a slightly asymmetric profile (width $\approx 200 \mathrm{~cm}^{-1}$ ) with an extended blue wing. The $5 d 6 s^{1} D_{2}$ state lies $\approx 1000 \mathrm{~cm}^{-1}$ below the $6 s 6 p^{3} P_{J}$ group. Laser excitation of $6 s 6 p^{3} P_{1}$ state populates the $5 d 6 s^{1} D_{2}$ state either by a (unobserved) long wavelength transition or a radiationless transfer, as evidenced by the observation of fluorescence at the $5 d 6 s{ }^{1} D_{2}-6 s^{2}{ }^{1} S_{0}$ transition.

\section{The long-lived metastable state $5 d 6 s{ }^{3} D_{1}$}

The forbidden $5 d 6 s^{3} D_{1}-6 s^{2}{ }^{1} S_{0}$ intercombination emission line at $9040 \mathrm{~cm}^{-1}$ is at the edge of our CCD detector's sensitivity range. Nevertheless, the corresponding fluorescence line can be observed in our experiments in solid He (Fig. 7). 


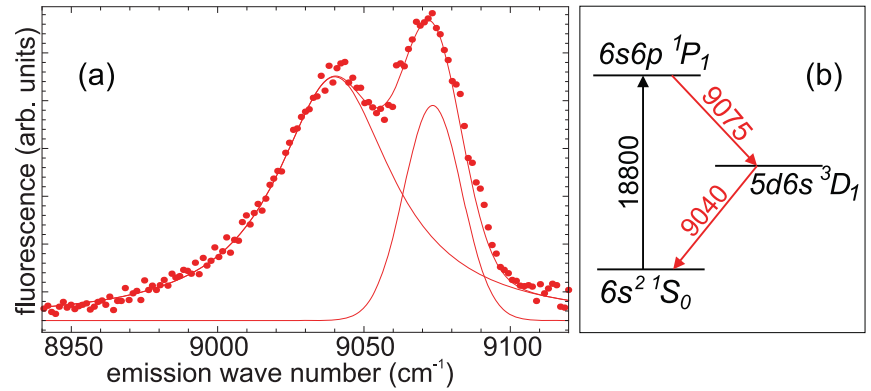

FIG. 7. (Color online) (a) Fluorescence spectra of the transitions between the $6 s^{2}{ }^{1} S_{0}, 6 s 6 p^{1} P_{1}$, and $5 d 6 s^{3} D_{1}$ states of Ba in solid $\mathrm{He}, 30$ bar, 1.5 K. Dots, experimental data; solid lines, Gaussian fits. (b) Relevant part of the energy diagram. Indicated wave numbers correspond to actual transitions in solid helium.

Its line center (width $\approx 30 \mathrm{~cm}^{-1}$ ) is practically not affected by the He matrix.

In Ba vapor experiments with noble buffer gas the lifetime of the $5 d 6 s^{3} D_{1}$ state is determined by quenching atomic collisions and reaches a several milliseconds [24]. In solid $\mathrm{He}$, however, we found that the lifetime of the $5 d 6 s^{3} D_{1}$ state is $2.72(4) \mathrm{s}$. We measured the lifetime by firing a train of $10-20$ Nd:YAG laser pulses at the sample, while recording a series of fluorescence spectra during and following the pulse train (Fig. 8). The resulting time dependence of the fluorescence intensity at $9040 \mathrm{~cm}^{-1}$ yields the decay curve (Fig. 8) of the metastable state population, from which the lifetime is extracted by an exponential fit.

We have identified an efficient population channel for this state. The wave number $\left(18800 \mathrm{~cm}^{-1}\right)$ of the Nd:YAG laser's second harmonic — used for ablation and cluster breakup in the present experiments (Sec. II) —overlaps with the blue wing of the strong $6 s^{2}{ }^{1} S_{0}-6 s 6 p{ }^{1} P_{1}$ resonance absorption line (Sec. III B). Population from the $6 s 6 p{ }^{1} P_{1}$ state decays via the $6 s 6 p^{1} P_{1}-6 s^{2}{ }^{1} S_{0}$ and the $6 s 6 p{ }^{1} P_{1}-5 d 6 s^{3} D_{1}$ transitions at 18115 and $9075 \mathrm{~cm}^{-1}$, respectively. The latter transition can be observed together with the fluorescence from the $5 d 6 s^{3} D_{1}$ state (to the ground state) at $9040 \mathrm{~cm}^{-1}$ (Fig. 7).

This mechanism leads to a large steady-state population of the metastable $5 d 6 s^{3} D_{1}$ state, absorption from which can be seen in the transmission spectrum of Fig. 1. Applying Eq. (1) to the absorption line shapes of the four transitions from the

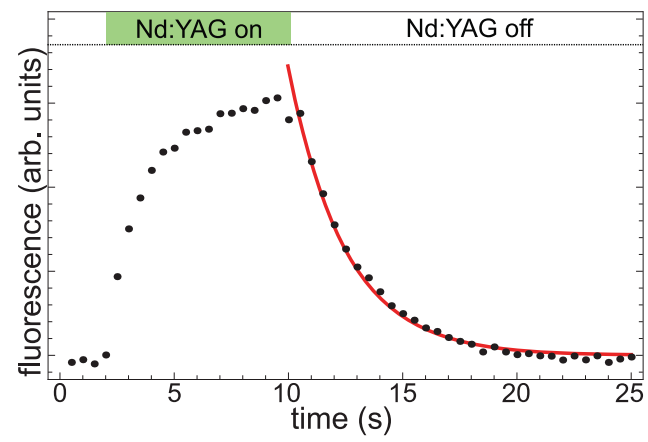

FIG. 8. (Color online) Fluorescence at $9040 \mathrm{~cm}^{-1}$ excited by a series of pulses of the frequency-doubled $\mathrm{Nd}$ :YAG laser $\left(18800 \mathrm{~cm}^{-1}\right)$.
TABLE III. Exponents $n$ of power-law fits $I(P) \propto P^{n}$ of the fluorescence intensities $I$ versus laser power $P$ of spectral lines excited by the second harmonic of Nd:YAG laser at $18800 \mathrm{~cm}^{-1}$.

\begin{tabular}{lcc}
\hline \hline \multicolumn{1}{c}{ Transition } & Wave number $\left(\mathrm{cm}^{-1}\right)$ & $n$ \\
\hline $5 d 6 p^{3} F_{2} \rightarrow 5 d 6 s^{3} D_{2}$ & 13810 & $1.59(4)$ \\
$5 d 6 p^{3} F_{1} \rightarrow 5 d 6 s^{3} D_{1}$ & 13150 & $1.52(7)$ \\
$5 d 6 p^{3} F_{1} \rightarrow 5 d 6 s^{3} D_{1}$ & 12970 & $1.56(5)$ \\
$6 s 7 s{ }^{3} S_{1} \rightarrow 6 s 6 p{ }^{3} P_{2}$ & 12690 & $1.20(3)$ \\
$\left(6 s 6 p^{3} P_{1} \rightarrow 6 s^{2}{ }^{1} S_{0}\right)$ & & \\
$5 d 6 s{ }^{1} D_{2} \rightarrow 6 s^{2}{ }^{1} S_{0}$ & 11390 & $0.84(4)$ \\
$5 d^{2}{ }^{3} P_{1} \rightarrow 6 s 6 p^{3} P_{0}$ & 11210 & $1.37(1)$ \\
$6 s 7 s{ }^{1} S_{0} \rightarrow 6 s 6 p{ }^{1} P_{1}$ & 10200 & $1.68(2)$ \\
$6 s 6 p{ }^{1} P_{1} \rightarrow 5 d 6 s^{3} D_{1}$ & 9075 & $1.0(3)$ \\
$6 s 6 p{ }^{1} P_{1} \rightarrow 6 s^{2}{ }^{1} S_{0}$ & 9040 & $0.94(3)$ \\
\hline \hline
\end{tabular}

${ }^{3} D_{1}$ state to the $5 d 6 p^{3} D_{1,2}$ and ${ }^{3} F_{2,3}$ states at 15740,16140 , 13500 , and $14445 \mathrm{~cm}^{-1}$, respectively, we obtain a metastable density of $N_{i}=1.3(3) \times 10^{14} \mathrm{~cm}^{-3}$, which is approximately $10 \%$ of the ground-state density. The ${ }^{3} D_{1}$ state serves as a ground state for the triplet transitions discussed in Sec. III E.

The two-step character of the excitation processes for some spectral lines is demonstrated by the dependence of their fluorescence yield, $I(P)$, on laser power $P$. We have fitted that dependence by a power law, $I(P) \propto P^{n}$ for the fluorescence lines that can be excited by frequency-doubled Nd:YAG laser $\left(18800 \mathrm{~cm}^{-1}\right)$ radiation. The results are shown in Table III. The transitions from one-photon excited states and two-photon excited states may be distinguished, since the former have an almost linear laser power dependence while the latter have an almost quadratic dependence. The fluorescence intensity, proportional to the excited state population, can be written as $I \propto \gamma_{\text {exc }} / \Gamma$, where $\gamma_{\text {exc }} \propto P^{n}$ is the (effective) $n$-photon excitation rate, and $\Gamma$ the population relaxation rate of the excited state. The power-law exponents $n$ for the $5 d 6 s^{1} D_{2}-$ $6 s^{2}{ }^{1} S_{0}\left(11390 \mathrm{~cm}^{-1}\right)$ and $5 d 6 s^{3} D_{1}-6 s^{2}{ }^{1} S_{0}\left(9040 \mathrm{~cm}^{-1}\right)$ fluorescence are 0.8(1) and 1.0(1), respectively, indicating a single-photon excitation. The power-law exponents for the fluorescence from the $5 d 6 p^{3} F_{J}$ states $(13810,13150$, and $12970 \mathrm{~cm}^{-1}$ ), which cannot be reached in single photon absorption, are equal to 1.59(4), 1.52(7), and 1.56(5), respectively. These states are thus not excited by a single photon transition.

The fact that the exponent $n$ is less than 2 for two-step excitations and less than 1 for direct excitation can be explained by assuming that the excited state's population loss rate, $\Gamma$, grows with the laser power. We attribute this effect to the softening of the helium host crystal due to laser heating and the ensuing increase of the Ba atoms' mobility. At elevated levels of the laser power it may explain the increase of $\Gamma$ with $P$ and hence the reduction of $n$.

\section{E. Triplet transitions $5 d 6 s-5 d 6 p$}

There exists a large number of strong transitions connecting the metastable $5 d 6 s{ }^{3} D_{J}$ states with the triplet states of the configuration $5 d 6 p$ (Fig. 10). These lines were studied by Bauer et al. [4] after the recombination of $\mathrm{Ba}^{+}$ions with free electrons injected into superfluid $\mathrm{He}$ at saturated vapor 
pressure. Laser-induced fluorescence of the same group of transitions was observed by Fujisaki et al. [7]. The lines observed in [4,7] are labeled in Table I with superscripts a and $b$, respectively. In agreement with the results of $[4,7]$, the fluorescence lines in liquid helium at low pressure are blueshifted by less than $25 \mathrm{~cm}^{-1}$ with respect to the free atomic wave numbers, and have a spectral width of $\approx 40 \mathrm{~cm}^{-1}$.

In our experiments, these fluorescence lines can be excited either by nonresonant frequency-tripled Nd:YAG laser radiation at $28195 \mathrm{~cm}^{-1}$ (Fig. 4), or by the signal beam of the OPO tuned into resonance with the corresponding absorption line. In the latter case the atoms are excited from the long-lived $5 d 6 s^{3} D_{1}$ state that is populated through the cluster breakup by the Nd:YAG laser at $18800 \mathrm{~cm}^{-1}$. Fujisaki et al. [7] have studied these absorption lines in superfluid $\mathrm{He}$ and found them to be more strongly broadened $\left(\mathrm{FWHM} \approx 195 \mathrm{~cm}^{-1}\right.$ ) and blueshifted $\left(\approx 180 \mathrm{~cm}^{-1}\right)$ than the fluorescence lines.

Our results show that - similar to the $6 s^{2}{ }^{1} S_{0}-6 s 6 p{ }^{1} P_{1}$ transition-the triplet lines have a larger shift and a stronger broadening in solid $\mathrm{He}$ than in low-pressure superfluid helium. Fluorescence (absorption) line shifts are typically $100(600) \mathrm{cm}^{-1}$ to the blue and the line widths are typically $\approx 60(300) \mathrm{cm}^{-1}$ (Table I).

Excitation and fluorescence spectra of the $5 d 6 s{ }^{3} D_{1,2,3}-$ $5 d 6 p^{3} P_{0,1,2}$ multiplet are shown in Fig. 9. The three $5 d 6 p^{3} P_{0,1,2}$ states are excited from the $5 d 6 s{ }^{3} D_{1}$ state via the corresponding absorption lines at 17 830, 17 620, and $17575 \mathrm{~cm}^{-1}$, respectively. The excitation lines are blueshifted by $\approx 600 \mathrm{~cm}^{-1}$ and their widths of $\approx 300 \mathrm{~cm}^{-1}$ are larger than the splittings between the upper states, so that their contours overlap.

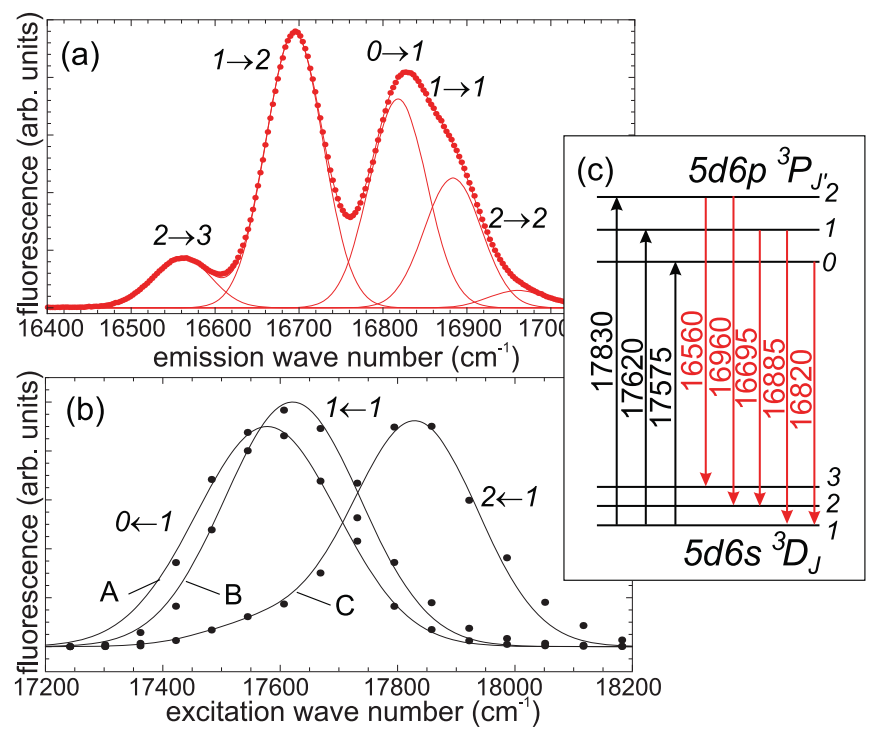

FIG. 9. (Color online) (a) Spectrum of the fluorescence from the $5 d 6 p^{3} P_{J^{\prime}}$ states toward the $5 d 6 s^{3} D_{J}$ states. Excitation laser at $17620 \mathrm{~cm}^{-1}, p=30 \mathrm{bar}, T=1.5 \mathrm{~K}$. (b) Excitation spectra of the $5 d 6 s^{3} D_{1}-5 d 6 p^{3} P_{J^{\prime}}$ transitions under the same conditions as (a). Curve A, fluorescence yield at $16820 \mathrm{~cm}^{-1}$; curve $\mathrm{B}$, fluorescence yield at $16695 \mathrm{~cm}^{-1}$; curve $C$, fluorescence yield at $16560 \mathrm{~cm}^{-1}$. (c) Relevant part of the energy diagram. Indicated wave numbers correspond to actual transitions in solid helium.

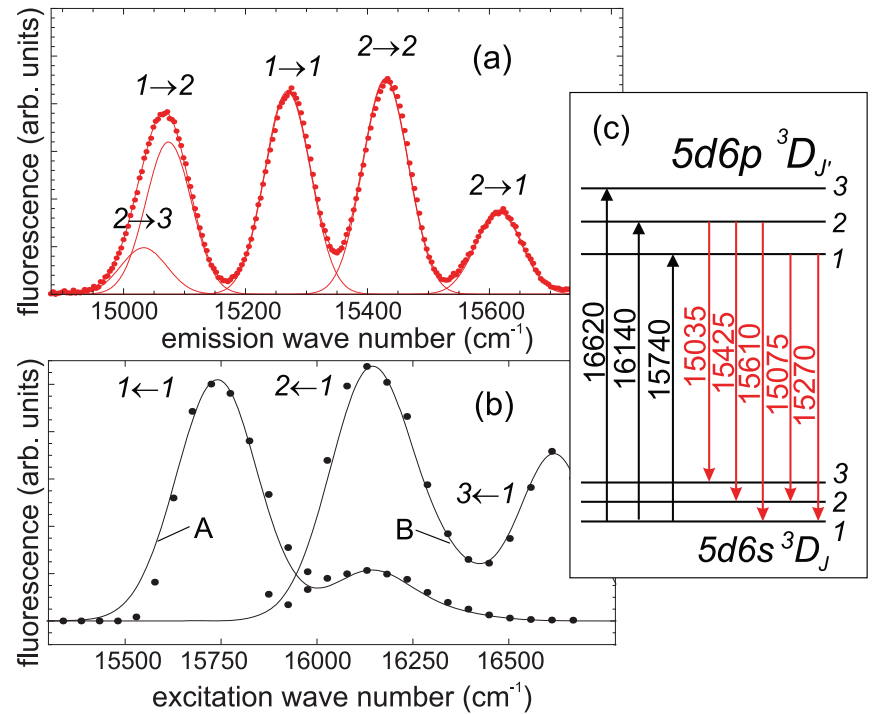

FIG. 10. (Color online) (a) Spectrum of the fluorescence from the $5 d 6 p^{3} D_{J^{\prime}}$ states toward the $5 d 6 s^{3} D_{J}$ states. Excitation laser at $16140 \mathrm{~cm}^{-1}, p=30$ bar, $T=1.5 \mathrm{~K}$. (b) Excitation spectra of the $5 d 6 s^{3} D_{1}-5 d 6 p^{3} D_{J^{\prime}}$ transitions under the same conditions as (a). Curve A, fluorescence yield at $15075 \mathrm{~cm}^{-1}$; curve B, fluorescence yield at $16620 \mathrm{~cm}^{-1}$. (c) Relevant part of the energy diagram. Indicated wave numbers correspond to actual transitions in solid helium.

The excitation and fluorescence spectra of the $5 d 6 s^{3} D_{1,2,3}-5 d 6 p{ }^{3} D_{1,2,3}$ multiplet are shown in Fig. 10. Similar to the ${ }^{3} P_{0,1,2}$ multiplet, the three $5 d 6 p{ }^{3} D_{1,2,3}$ states are excited from the $5 d 6 s^{3} D_{1}$ state via the corresponding absorption lines at 16620,16140 , and $15740 \mathrm{~cm}^{-1}$, respectively. The excitation lines are blueshifted by $\approx 650 \mathrm{~cm}^{-1}$ and their width is $\approx 300 \mathrm{~cm}^{-1}$, so that there is practically no line overlap. However, excitation of the upper ${ }^{3} D_{3}$ state also induces fluorescence from the two lower-lying ${ }^{3} D_{1,2}$ states of the multiplet. Similarly, excitation of the ${ }^{3} D_{2}$ state also induces fluorescence from the lowest ${ }^{3} D_{1}$ state. Moreover, fluorescence from the $5 d 6 p^{3} D_{J}$ multiplet can be induced as well by laser excitation of the higher-lying $5 d 6 p{ }^{3} P$ states. The helium matrix thus efficiently induces inter- and intramultiplet mixing of states.

Excitation and fluorescence spectra of the $5 d 6 s{ }^{3} D_{1,2,3}-$ $5 d 6 p^{3} F_{2,3,4}$ transitions are shown in Fig. 11. Transitions from the $5 d 6 s{ }^{3} D_{1}$ state to the $5 d 6 p^{3} F_{4}$ state are forbidden by angular momentum conservation, so that the ${ }^{3} F_{4}$ state cannot be excited directly. The $5 d 6 p^{3} F_{3}$ state can be excited from the $5 d 6 s^{3} D_{1}$ state via absorption on the $5 d 6 s^{3} D_{1}-5 d 6 p^{3} F_{3}$ transition at $14445 \mathrm{~cm}^{-1}$. That excitation line is blueshifted by $640 \mathrm{~cm}^{-1}$ and is $\approx 300 \mathrm{~cm}^{-1}$ broad. Finally, the transition to the lowest ${ }^{3} F_{J}$ state, $5 d 6 p^{3} F_{2}$, could not be excited resonantly because its wavelength (estimated to be at $\approx 13800 \mathrm{~cm}^{-1}$ ) is outside the tuning range of our OPO. However, the state can be populated by a radiationless transition from the $900 \mathrm{~cm}^{-1}$ higher-lying $5 d 6 p^{3} F_{3}$ state. We have also observed a second population channel of the $5 d 6 p^{3} F_{2,3}$ states involving absorption from the $5 d 6 s^{3} D_{1}$ metastable state to the singlet $5 d 6 p{ }^{1} D_{2}$ state that is nearly degenerate and thus readily mixed with the $5 d 6 p{ }^{3} F_{3}$ state. Last, but not least, there is an efficient population transfer to the $5 d 6 p^{3} F_{2,3}$ states from the higher-lying $5 d 6 p{ }^{3} \mathrm{D}$ multiplet [Fig. 11(b)]. 


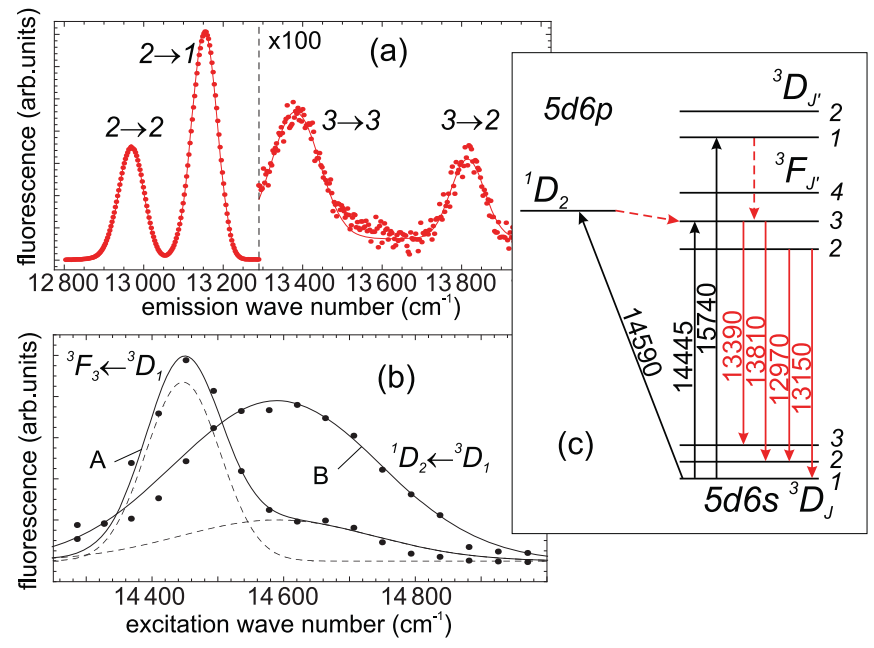

FIG. 11. (Color online) (a) Spectrum of the fluorescence from the $5 d 6 p^{3} F_{J^{\prime}}$ states toward the $5 d 6 s^{3} D_{J}$ states. Excitation laser at $15740 \mathrm{~cm}^{-1}, p=30$ bar, $T=1.5 \mathrm{~K}$. (b) Excitation spectra of the $5 d 6 s^{3} D_{1}-5 d 6 p^{3} F_{J^{\prime}}$ transitions under the same conditions as (a). Curve A, fluorescence yield at $13810 \mathrm{~cm}^{-1}$; curve B, fluorescence yield at $13150 \mathrm{~cm}^{-1}$. (c) Relevant part of the energy diagram. Indicated wave numbers correspond to actual transitions in solid helium.

\section{F. Population transfer}

As discussed earlier in Sec. IIID, the absorption of the second harmonic of $\mathrm{Nd}$ :YAG radiation by ground-state $\mathrm{Ba}$ atoms leads to a very efficient population of the long-lived $5 d 6 s^{3} D_{1}$ state, from which further excitation to the triplet $5 d 6 p$ states is possible (see Sec. IIIE). By using a pulse sequence of frequency-doubled Nd:YAG radiation at $18800 \mathrm{~cm}^{-1}$, together with OPO pulses at $15740 \mathrm{~cm}^{-1}$, we were able to measure the metastable population dynamics by a method that circumvents the recording of weak fluorescence from the forbidden $5 d 6 s^{3} D_{1}-6 s^{2}{ }^{1} S_{0}$ deexcitation channel (Fig. 12).

The OPO - firing at a rate of $10 \mathrm{~Hz}$ - is tuned to be resonant with the $5 d 6 s^{3} D_{1}-5 d 6 p^{3} D_{1}$ absorption line at $15740 \mathrm{~cm}^{-1}$. We record the fluorescence spectra of the strong $5 d 6 p{ }^{3} F_{2}-$ $5 d 6 s^{3} D_{1,2}$ doublet at $13150 / 12970 \mathrm{~cm}^{-1}$ with an exposure time of $50 \mathrm{~ms}$ following each OPO pulse. The Nd:YAG laser fires a train of 30 pulses at the same rate during $3 \mathrm{~s}$. The resulting time dependence of the OPO-induced fluorescence intensity is shown in Fig 13. The rise and the decay of the fluorescence signal are both governed by the slow decay of the intermediate metastable state. Similar measurements were done with the OPO tuned to the $5 d 6 s^{3} D_{1}-5 d 6 p{ }^{3} D_{0}$ and $5 d 6 s^{3} D_{1}-5 d 6{ }^{3} F_{3}$ transitions at 15740 and $14445 \mathrm{~cm}^{-1}$, respectively.

The resulting $5 d 6 s^{3} D_{1}$ lifetimes vary from 0.9 to $2.6 \mathrm{~s}$, depending on the OPO wavelength and intensity, and are thus shorter than the $2.6 \mathrm{~s}$ reported in Sec. III D. This observation suggests the existence of an additional decay channel that depopulates the $5 d 6 s^{3} D_{1}$ state, which is not fully repopulated after the OPO excitation to the $5 d 6 p^{3} D_{3}$ state. As discussed in Sec. III E, excitation of the $5 d 6 p^{3} D_{1}$ state is partly transferred to lower-lying $5 d 6 p^{3} F_{J}$ states by far infrared radiation or radiationless transitions. Within the $5 d 6 p^{3} F_{J}$ states there is also a population transfer to the lowest $J=1$ state. We have

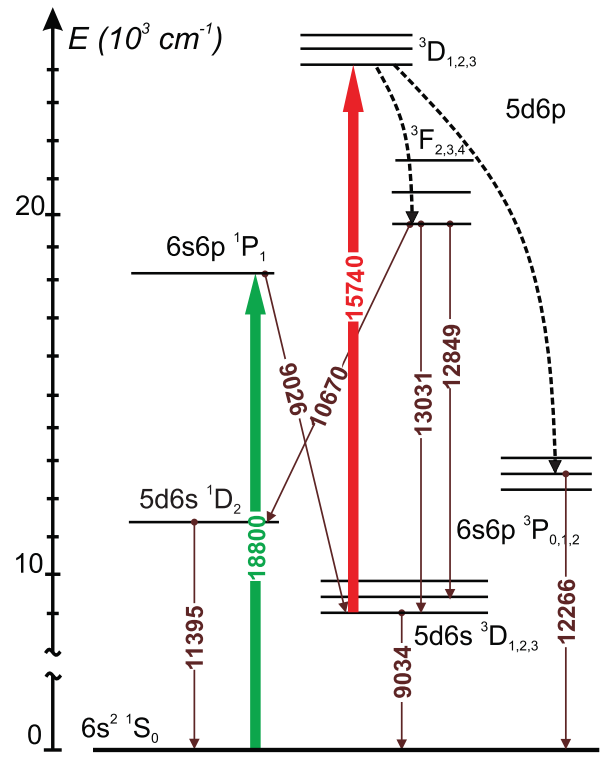

FIG. 12. (Color online) Two-step excitation and population transfer. Green and red thick arrows indicate transitions excited with the Nd:YAG laser and OPO, respectively. Dashed arrows indicate (radiationless) transitions. Solid brown arrows indicate fluorescence transitions, observed in the experiment.

observed at least one additional channel of radiative decay of the $5 d 6 p{ }^{3} F_{2}$ state, viz., a transition to the singlet $5 d 6 s{ }^{1} D_{2}$ state at $10800 \mathrm{~cm}^{-1}$ (in solid helium). Similar transitions may occur toward the triplet $6 s 6 p^{3} P_{J}$ states with wavelengths between 8000 and $10000 \mathrm{~cm}^{-1}$. We have indeed observed a weak fluorescence line at $9800 \mathrm{~cm}^{-1}$ under similar excitation at $18800 \mathrm{~cm}^{-1}$ that may correspond to the $5 d 6 p^{3} F_{2}-6 s 6 p^{3} P_{0}$ transition. Finally, we can observe fluorescence (at 11390 and $12640 \mathrm{~cm}^{-1}$, respectively) from these lower-lying $5 \mathrm{~d} 6 \mathrm{~s}^{1} \mathrm{D}_{2}$ and $6 s 6 p{ }^{3} P_{1}$ quasi-metastable states. These two fluorescence lines exhibit exactly the same dynamics as discussed above, with a slow rise and decay determined by the lifetime of the $5 d 6 s{ }^{3} D_{1}$ state in these two-step experiments. It is thus the efficient population transfer from the triplet $5 d 6 p$ states to related two low-lying states that is responsible for the observed shortening of the $5 d 6 s^{3} D_{1}$ lifetime.

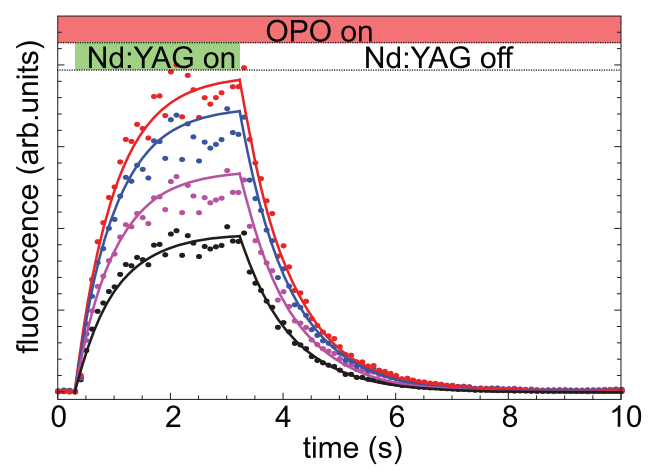

FIG. 13. (Color online) Dynamics of fluorescence excited via two-step OPO + Nd:YAG absorption. From top to bottom: red, $5 d 6 p^{3} F_{2}-5 d 6 s^{3} D_{1}$ transition; blue, $5 d 6 p^{3} F_{2}-5 d 6 s^{3} D_{2}$ transition; magenta, $6 s 6 p^{3} P_{1}-6 s^{2}{ }^{1} S_{0}$ transition; black, $5 d 6 s{ }^{1} D_{2}-$ $6 s^{2}{ }^{1} S_{0}$ transition. 


\section{G. Unidentified spectral lines}

Some of the observed spectral features cannot be unambiguously identified. The fluorescence line at $17350 \mathrm{~cm}^{-1}$ could be excited by the second and the third harmonics of the Nd:YAG laser (Fig. 4) and by the OPO at 16 140, 15 740, and $14445 \mathrm{~cm}^{-1}$. These three absorption lines have been reliably attributed to the triplet $5 d 6 s-5 d 6 p$ transitions (Sec. III E). One thus expects that fluorescence at $17350 \mathrm{~cm}^{-1}$ originates from a state that can be populated via the triplet $5 d 6 p$ states. Several fluorescence transitions of the free $\mathrm{Ba}$ atom have wave numbers close to $17350 \mathrm{~cm}^{-1}: 5 d 6 p{ }^{1} P_{1}-5 d 6{ }^{1} D_{2}$ (17 $\left.159 \mathrm{~cm}^{-1}\right), 6 s 6 d^{3} D_{1,2,3}-6 s 6 p^{3} P_{1}$ (17 091, 17 236, $\left.17303 \mathrm{~cm}^{-1}\right), 6 p^{2}{ }^{3} P_{2}-6 s 6 p{ }^{1} P_{1}\left(17284 \mathrm{~cm}^{-1}\right), 5 d 6 p{ }^{1} F_{3}-$ $5 d 6 s^{3} D_{3}\left(17219 \mathrm{~cm}^{-1}\right)$. However, the upper states of all these lines lie above the triplet $5 d 6 p$ states (Fig. 3 ) and can therefore not be populated by a radiationless or fluorescence transition from any of them. Absorption of one more photon from the OPO or from the declusterization laser $\left(18800 \mathrm{~cm}^{-1}\right)$ is thus required.

Another unidentified fluorescence line lies at $10420 \mathrm{~cm}^{-1}$. It can be excited only by frequency-doubled and -tripled Nd:YAG laser (18 800 and $28195 \mathrm{~cm}^{-1}$ ).

\section{SUMMARY}

We have observed LIF spectra of barium atoms implanted in liquid and solid helium matrices. Due to the very large density of implanted atoms, we have found a number of spectral lines that have not been observed before in liquid and solid helium matrices. We have measured the lifetimes of the $5 d 6 s{ }^{3} D_{1}$ and $6 s 6 p^{3} P_{1}$ metastable states that are found to be $2.6 \mathrm{~s}$ and $1.2 \mu \mathrm{s}$, respectively. The laser-induced preparation of long-lived metastable states provides highly efficient channels for two-step excitations of high-lying states that are inaccessible by direct absorption from the ground state. We have also observed radiationless inter- and intramultiplet population transfers that are induced by the solid He matrix. The very high densities of $\mathrm{Ba}$ atoms that can be achieved in solid helium matrices make those dopants the most convenient species known today for studying dopant-matrix interactions as well as the structure and dynamics of the helium matrix itself. Recent advances in the development of ab initio Ba-He interaction potentials in the ground and excited states [25-27] open possibilities that did not exist in the early 1990s, for the quantitative modeling of superfluid and solid quantum matrix-isolated barium atoms. Such model calculations have been recently performed for the $\mathrm{Ba}$ atoms on helium droplets [28]. The extensive spectroscopic data provided here will prove useful for testing the modeling of the $\mathrm{Ba}-\mathrm{He}$ interaction in bulk matrices.

\section{ACKNOWLEDGMENT}

This work was supported by Grant No. 200020-129831 of the Schweizerischer Nationalfonds.
[1] P. Moroshkin, A. Hofer, and A. Weis, Phys. Rep. 469, 1 (2008).

[2] M. Himbert, A. Lezama, and J. Dupont-Roc, J. Phys. 46, 2009 (1985).

[3] H. J. Reyher, H. Bauer, C. Huber, R. Mayer, A. Schäfer, and A. Winnacker, Phys. Lett. A 115, 238 (1986).

[4] H. Bauer, M. Beau, A. Bernhardt, B. Friedl, and H. J. Reyher, Phys. Lett. A 137, 217 (1989).

[5] H. Bauer, M. Beau, B. Friedl, C. Marchand, K. Miltner, and H. J. Reyher, Phys. Lett. A 146, 134 (1990).

[6] B. Tabbert, M. Beau, H. Günther, W. Haussler, C. Hönninger, K. Meyer, B. Plagemann, and G. zu Putlitz, Z. Phys. B 97, 425 (1995).

[7] A. Fujisaki, K. Sano, T. Kinoshita, Y. Takahashi, and T. Yabuzaki, Phys. Rev. Lett. 71, 1039 (1993).

[8] J. H. M. Beijersbergen, Q. Hui, and M. Takami, Phys. Lett. A 181, 393 (1993).

[9] Q. Hui, J. L. Persson, J. H. M. Beijersbergen, and M. Takami, Z. Phys. B 98, 353 (1995).

[10] S. I. Kanorsky, M. Arndt, R. Dziewior, A. Weis, and T. W. Hänsch, Phys. Rev. B 50, 6296 (1994).

[11] S. I. Kanorsky, M. Arndt, R. Dziewior, A. Weis, and T. W. Hänsch, Phys. Rev. B 49, 3645 (1994).

[12] J. Jortner, W. R. Kestner, S. A. Rice, and M. H. Cohen, J. Chem. Phys. 43, 2614 (1965).

[13] M. Beau, H. Günther, G. zu Putlitz, and B. Tabbert, Z. Phys. B 101, 253 (1996).

[14] J.-Z. Tang, M. Kimura, and I. Shimamura, Chem. Phys. Lett. 256, 327 (1996).
[15] P. Moroshkin, A. Hofer, S. Ulzega, and A. Weis, Nat. Phys. 3, 786 (2007).

[16] P. Moroshkin, V. Lebedev, and A. Weis, Phys. Rev. Lett. 102, 115301 (2009).

[17] M. Arndt, Ph.D. thesis, Ludwig Maximilians Universität München, 1995, Max-Planck Institut für Quantenoptik, Garching, MPQ-Report 197.

[18] T. Eichler, R. Müller-Siebert, D. Nettels, S. Kanorsky, and A. Weis, Phys. Rev. Lett. 88, 123002 (2002).

[19] Y. Fukuyama, Y. Moriwaki, and Y. Matsuo, Phys. Rev. A 69, 042505 (2004).

[20] V. Lebedev, P. Moroshkin, and A. Weis, J. Phys. Chem. A 115, 7169 (2011).

[21] L. Jahreiss and M. C. E. Huber, Phys. Rev. A 31, 692 (1985).

[22] S. Niggli and M. C. E. Huber, Phys. Rev. A 35, 2908 (1987).

[23] N. D. Scielzo, J. R. Guest, E. C. Schulte, I. Ahmad, K. Bailey, D. L. Bowers, R. J. Holt, Z.-T. Lu, T. P. O'Connor, and D. H. Potterveld, Phys. Rev. A 73, 010501 (2006).

[24] J. W. Adams, J. Lei, and D. Husain, Z. Phys. Chem. 215, 1205 (2001).

[25] E. Czuchaj, F. Rebentrost, H. Stoll, and H. Preuss, Chem. Phys. 196, 37 (1995).

[26] J. Brust and C. H. Greene, Phys. Rev. A 56, 2005 (1997).

[27] C. C. Lovallo and M. Klobukowski, J. Chem. Phys. 120, 246 (2004).

[28] A. Hernando, R. Mayol, M. Pi, M. Barranco, F. Ancilotto, O. Bünermann, and F. Stienkemeier, J. Phys. Chem. A 111, 7303 (2007). 